\title{
Integral equation theory for two-dimensional polymer melts
}

\author{
Arun Yethiraj and Bong June Sung \\ Theoretical Chemistry Institute and Department of Chemistry, University of Wisconsin, \\ Madison, Wisconsin 53706 \\ Fred Lado \\ Department of Physics, North Carolina State University, Raleigh, North Carolina 27695
}

(Received 30 July 2004; accepted 15 December 2004; published online 1 March 2005)

\begin{abstract}
The polymer reference interaction site model theory is investigated for two-dimensional polymer melts composed of freely-jointed hard disk chains and tangent-disk rods. Exact results for the intramolecular pair correlation functions are input into the theory, and predictions of the theory for the intermolecular pair correlation functions are tested via comparison with simulation. The theory is not as accurate for this system as it is for three-dimensional polymer melts, and the quantitative predictions are not good except at the highest area fractions. Possible reasons for the deficiency in the theory are discussed. (C) 2005 American Institute of Physics. [DOI: 10.1063/1.1857520]
\end{abstract}

\section{INTRODUCTION}

The behavior of confined polymer melts is a subject of current interest. ${ }^{1}$ An extreme case occurs when the confinement is severe enough that the molecules can be considered two dimensional. Such a situation is experimentally realized when DNA molecules are adsorbed to charged lipid bilayers. ${ }^{2}$ In a recent paper, ${ }^{3}$ one of us presented computer simulation results for the static properties of a tangent hard disk model of two-dimensional polymers. There were many surprising results that suggested that long-standing scaling ideas $^{4,5}$ might require reconsideration. In this paper, we investigate the polymer reference interaction site model (PRISM) theory for two-dimensional polymer solutions and melts.

There has been considerable research on the PRISM theory for polymers in three dimensions. ${ }^{6}$ The RISM theory ${ }^{7}$ consists of a set of nonlinear integral equations that provide a relationship between the site-site intermolecular direct correlation functions, the site-site intramolecular correlation functions, and the site-site total correlation functions. If the intramolecular correlation functions are known, the RISM equations can be solved for the intermolecular correlation functions, given an approximate closure relation. The PRISM equations are obtained if end effects are ignored and all the sites on a molecule are treated as equivalent. For polymer melts in three dimensions, the venerable PercusYevick (PY) closure is accurate for the pair correlation functions in hard chains. To our knowledge there has been no investigation of the PRISM theory in two dimensions.

In this work, we test the PRISM theory for the pair correlation functions in two-dimensional polymers. We consider two models: freely-jointed tangent-disk chains and tangentdisk rods. We use exact results (obtained from simulations ${ }^{3}$ for flexible chains) for the intramolecular correlation functions and compare the predicted intermolecular correlation functions to simulation results. This comparison constitutes a test of the PRISM theory and closures since the intramolecular correlations are exact. We find that the theory is not as accurate as the corresponding theory for three-dimensional polymers. For flexible chains, at low densities the theory significantly overestimates the value of the average intermolecular pair correlation function, $g(r)$, at all distances. At high densities the theory is accurate for $r>1.5 \sigma$, where $\sigma$ is the disk diameter, but overestimates the value of $g(r)$ for shorter distances by about $50 \%$. For fluids of diatomic molecules, the theory overestimates the value of $g(r)$ at contact at low densities, but is quite accurate at high densities. For longer rods, at low densities the theory is in qualitative agreement with simulations although it tends to overestimate the value of $g(r)$ near contact. At high densities, however, the theory fails to reproduce some qualitative features in $g(r)$ such as the location of cusps.

The rest of the paper is organized as follows. The theory is presented in Sec. II, simulations for rods are described in Sec. III, a comparison between theory and simulations are presented in Sec. IV, and some conclusions are presented in Sec. V.

\section{POLYMER RISM THEORY}

The RISM theory of Chandler and Andersen ${ }^{7}$ provides a relationship between the site-site direct correlation functions, $c_{\alpha \beta}(r)$, the site-site intramolecular correlation functions, $\omega_{\alpha \beta}(r)$, and the site-site intermolecular correlation functions, $h_{\alpha \beta}(r)$, between sites $\alpha$ and $\beta$. In Fourier space, the RISM equations can be written as

$$
\hat{h}_{\alpha \beta}(k)=\sum_{\gamma, \delta=1}^{N} \hat{\omega}_{\alpha \gamma}(k) \hat{c}_{\gamma \delta}(k)\left[\hat{\omega}_{\delta \beta}(k)+\rho \hat{h}_{\delta \beta}(k)\right],
$$

where $N$ is the number of sites, $\rho$ is the number density of molecules, carets denote Fourier transforms, and $k$ is the momentum transfer variable. For two-dimensional systems, the Fourier transform pair is defined as

$$
f(r)=\frac{1}{2 \pi} \int_{0}^{\infty} k \hat{f}(k) J_{0}(k r) d k
$$




$$
\hat{f}(k)=2 \pi \int_{0}^{\infty} r f(r) J_{0}(k r) d r,
$$

where $J_{0}(x)$ is the zeroth-order Bessel function of the first kind, with the representation

$$
J_{0}(x)=\frac{1}{2 \pi} \int_{0}^{2 \pi} d \theta \exp (i x \cos \theta) .
$$

Given closure relations between $h_{\alpha \beta}(r)$ and $c_{\alpha \beta}(r)$, the RISM equations can be solved for the correlation functions. For hard chain polymers, the PY closure approximation is

$$
\begin{array}{ll}
g_{\alpha \beta}(r)=0, & r<\sigma_{\alpha \beta}, \\
c_{\alpha \beta}(r)=0, & r>\sigma_{\alpha \beta},
\end{array}
$$

where $\sigma_{\alpha \beta}$ is the distance of closest approach of sites $\alpha$ and $\beta$.

The polymer RISM theory is obtained by treating some of the sites as equivalent and averaging over them. If all the sites are treated as equivalent, the polymer RISM equation takes the form

$$
\hat{h}(k)=\hat{\omega}(k) \hat{c}(k) \hat{\omega}(k)+\rho_{m} \hat{\omega}(k) \hat{c}(k) \hat{h}(k),
$$

where $\rho_{m}=N \rho, \hat{h}(k)=\left(1 / N^{2}\right) \sum_{i j} \hat{h}_{i j}(k), \hat{c}(k)=\left(1 / N^{2}\right) \sum_{i j} \hat{c}_{i j}(k)$, and $\hat{\omega}(k)=(1 / N) \sum_{i j} \hat{\omega}_{i j}(k)$. It is often convenient to divide the sites on the molecule into subsets, with all sites of each subset treated as equivalent. An example is rods where the end sites are treated differently from the middle sites, with all the latter being treated as equivalent. In this case the PRISM equations take the form ${ }^{8,9}$

$$
\hat{\mathbf{H}}=\hat{\mathbf{\Omega}} \hat{\mathbf{C}} \hat{\mathbf{\Omega}}+\hat{\mathbf{C}} \hat{\mathbf{\Omega}} \hat{\mathbf{H}},
$$

where $\hat{\mathbf{H}}, \hat{\mathbf{C}}$, and $\hat{\mathbf{\Omega}}$ are matrices of Fourier transforms of the total, direct, and intramolecular correlation functions, respectively. The elements of the $\hat{\mathbf{H}}$ matrix are given by $\hat{H}_{M M^{\prime}}(k)$ $=\rho_{M} \rho_{M^{\prime}} \hat{h}_{M M^{\prime}}(k)$, where $\rho_{i}$ is the number density of sites of type $i, h_{M M^{\prime}}(r)\left[c_{M M^{\prime}}(r)\right]$ is the total (direct) correlation function between sites of type $M$ and sites of type $M^{\prime}$, and the elements of $\hat{\boldsymbol{\Omega}}$ are $\hat{\Omega}_{M M^{\prime}}(k)=\rho \Sigma_{\alpha=1}^{N_{M}} \sum_{\gamma=1}^{N_{M^{\prime}}} \hat{\omega}_{\alpha \gamma}(k)$, where $\alpha \in M, \gamma \in M^{\prime}$, and $N_{i}$ is the number of sites of type $i$.

The integral equations are solved iteratively using a Picard iteration scheme, ${ }^{10}$ described below for the case of the PRISM theory. We start with an initial guess for $c(r)$ and evaluate the Fourier transform using a numerical Hankel transform routine. The function $\hat{\gamma}(k) \equiv \hat{h}(k)-\hat{c}(k)$ is then evaluated using the PRISM equation, and inverted to give $\gamma(r)$. The next guess for $c(r)$ is then obtained from the closure relation. The PY closure takes the form

$$
\begin{aligned}
& c(r)=-1-\gamma(r), \quad r<\sigma, \\
& c(r)=0, \quad r>\sigma,
\end{aligned}
$$

and the hypernetted chain (HNC) closure takes the form

$$
\begin{aligned}
& c(r)=-1-\gamma(r), \quad r<\sigma, \\
& c(r)=e^{\gamma(r)}-1-\gamma(r), \quad r>\sigma .
\end{aligned}
$$

The functions are discretized in real space between $r=0$ and $R$, with $M$ unevenly spaced points chosen so that $r_{i}=\mu_{i} R / \mu_{M}$ where $\mu_{j}$ is the $j$ th positive root of $J_{0}(x)$. The results are insensitive to $R$ and $M$ if these parameters are large enough; in this work, we choose $R=50 \sigma$ and $M=1000$. The discrete Fourier transforms take the form ${ }^{11}$

$$
\begin{aligned}
& f\left(r_{i}\right)=\frac{1}{2 \pi} \sum_{j=1}^{M-1} \Delta k_{j} k_{j} \hat{f}\left(k_{j}\right) J_{0}\left(k_{j} r_{i}\right), \\
& \hat{f}\left(k_{j}\right)=2 \pi \sum_{i=1}^{M-1} \Delta r_{i} r_{i} f\left(r_{i}\right) J_{0}\left(k_{j} r_{i}\right),
\end{aligned}
$$

where

$$
\begin{aligned}
& \Delta r_{i}=\frac{2}{K^{2} r_{i}\left[J_{0}^{\prime}\left(k_{M} r_{i}\right)\right]^{2}}, \\
& \Delta k_{j}=\frac{2}{R^{2} k_{j}\left[J_{0}^{\prime}\left(k_{j} R\right)\right]^{2}},
\end{aligned}
$$

$J_{0}^{\prime}(x)=d J_{0}(x) / d x, k_{j}=\mu_{j} / R$, and $K=k_{N}$. The above method is a slow Fourier transform but satisfies the orthogonality conditions, ${ }^{11}$ which are important because the functions are transformed repeatedly.

\section{MONTE CARLO SIMULATIONS}

To investigate the effect of molecular geometry on the performance of the theory we perform simulations for tangent-disk rods where each molecule is composed of $N$ tangent disks arranged in a linear fashion. The simulation method is similar to that used earlier for flexible chains. ${ }^{3}$ The simulation cell is a square with periodic boundary conditions in all directions. For each area fraction we perform simulations with 100 and 500 molecules; no finite size effects are observed. Initial configurations are generated using a growth and equilibration algorithm. In the course of a simulation, molecules are moved using either reptation, translation, or rotation, chosen with equal probability. The simulation is a standard application of the Metropolis algorithm in the canonical ensemble where moves are accepted if the new configuration is free of overlap, and rejected otherwise. The system is equilibrated and properties are averaged over 10 runs of 1 million attempted moves each. We restrict our study to short rods, i.e., $N=2,4$, and 8 , because longer rods form nematic phases at low densities ${ }^{12}$ which the integral equation theories cannot predict. In each case we perform simulations for area fractions, $\phi$ (defined as $\phi=\pi \rho N \sigma^{2} / 4$ ) of $0.1,0.3$, and 0.5 . Figure 1 depicts snapshots of final configurations for $N=8$, and $\phi=0.1$ and 0.5 , respectively. The systems are clearly isotropic, although there is significant local orientational order at the higher area fraction.

\section{RESULTS AND DISCUSSION}

Theoretical predictions for $g(r)$ in freely-jointed chains from the PRISM theory (with the PY closure) are compared to simulation results ${ }^{3}$ in Figs. 2(a) and 2(b) for $N=16$ and 64, respectively. At low area fractions, the theory significantly 


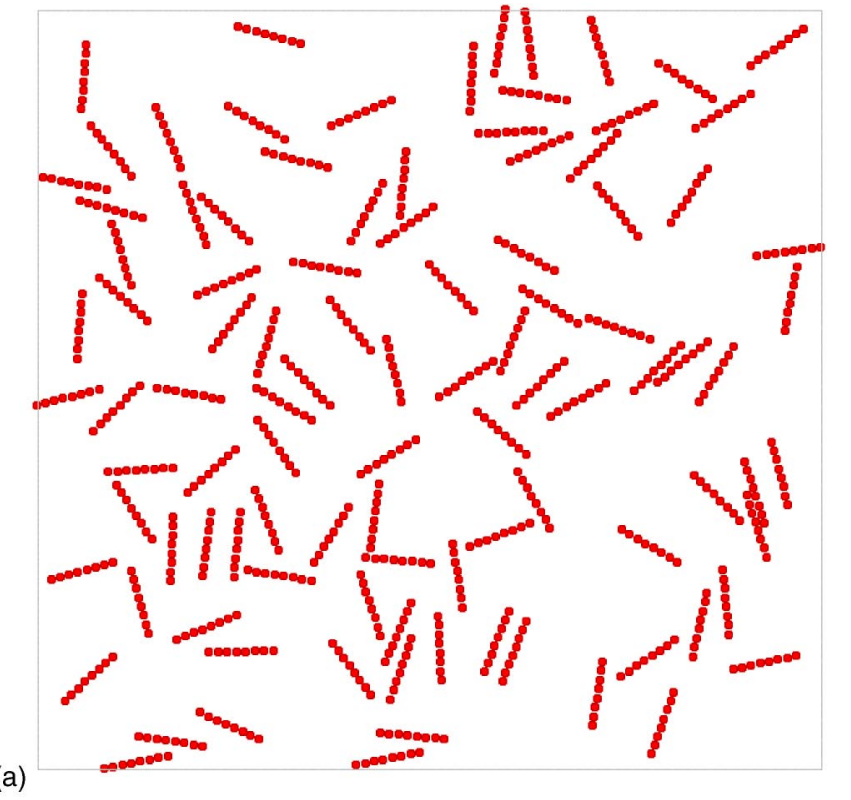

(a)

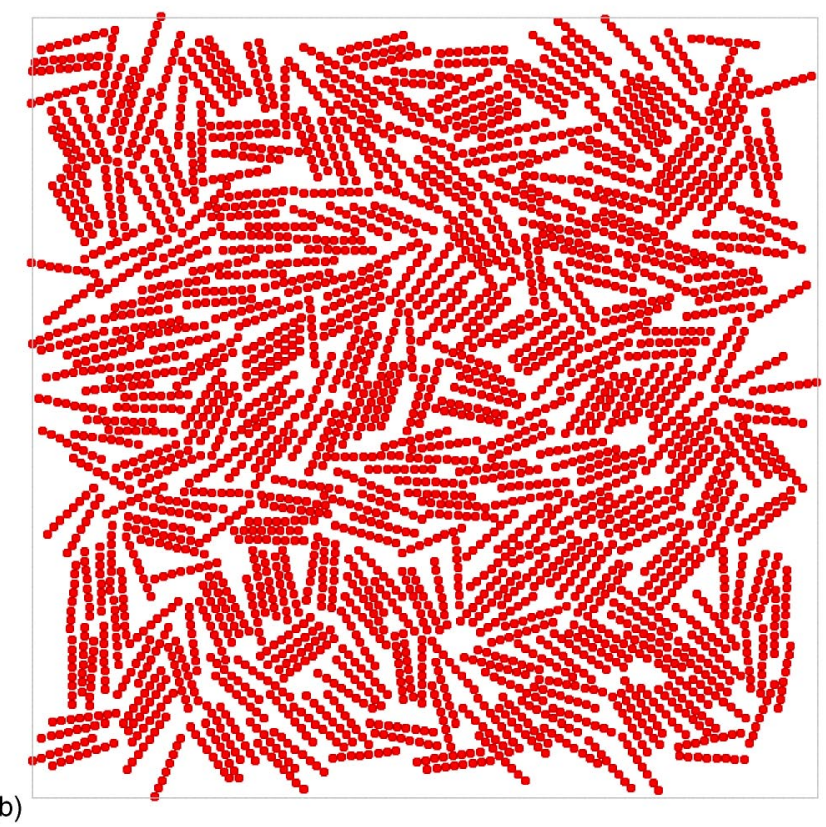

FIG. 1. Snapshots of final configurations for $N=8$ rods for (a) $\phi=0.1$ and (b) $\phi=0.5$.

overestimates the value of $g(r)$ over the entire range for which simulations are available. As the area fraction is increased, the agreement between theory and simulation improves, and at high area fractions $(\phi=0.5)$ the theory is in good agreement with the simulations for $r>1.5 \sigma$. For smaller distances the theory again significantly overestimates the value of $g(r)$. The performance of the theory is similar for all chain lengths studied from $N=16$ to 128 (two chain lengths are shown in Fig 2). The overestimation of $g(r)$ near contact by PRISM is also seen in three-dimensional polymer fluids, although it is not as severe as in the two-dimensional case. At high densities, PRISM is much more accurate in three dimensions than it is in two dimensions.

The theory is quite accurate for diatomics at high densities, but the accuracy diminishes for longer rods. Figures 3(a)-3(c) compare PRISM predictions (solid lines) for rods
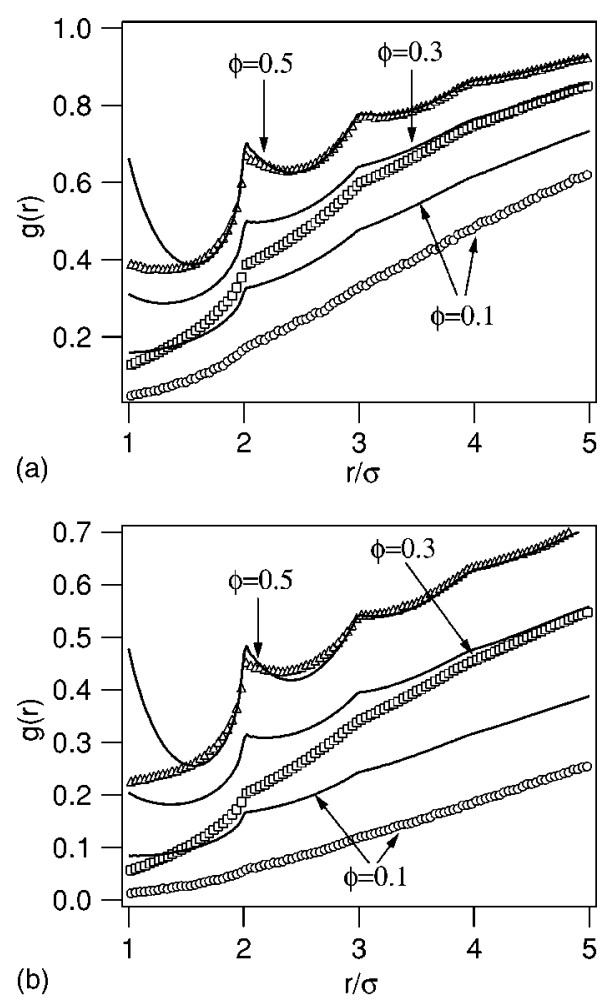

FIG. 2. Comparison of theoretical predictions for $g(r)$ (lines) to Monte Carlo simulations (Ref. 3) (symbols) of flexible chains, for various area fractions, and for (a) $N=16$ and (b) $N=64$.

to Monte Carlo simulation results for $N=2,4$, and 8, respectively. Note that for $N=2$ RISM and PRISM are identical (since all sites are equivalent) and for $N=4$ there are only two types of nonequivalent sites (end sites and middle sites). At low area fractions, large depletion effects are observed in the simulations, with values of $g(r)$ less than 1 . For these state points PRISM tends to overestimate the value of $g(r)$ as is also seen for flexible chains. For fluids of diatomic molecules, at high densities there is a second peak at $r=2 \sigma$ followed by hard-disk-like layering at larger distances. The RISM theory is in excellent agreement with simulations of diatomics for $\phi=0.5$. For fluids of longer rods, at high area fractions, there is considerable structure in $g(r)$ with a peak at short distances, followed by peaks at $r=(\sqrt{3}+j) \sigma$ and $r=j \sigma$ for integer $j$. The predictions of the PRISM theory are quite poor when compared to the simulations. The shape of $g(r)$ in the theory is quite different from the simulations and the location of the peaks is not predicted correctly, as is also seen in three dimensions. ${ }^{13}$

Relaxing the equivalent site approximation in the PRISM theory results in an improvement in the theoretical predictions at low densities. For dimers, the equivalent site approximation is exact and the PRISM theory is identical to the RISM theory. The success of the theory for this case suggests that we test the equivalent site approximation inherent to the PRISM theory. The dashed lines in Fig. 3(b) are predictions of the RISM theory, which are more accurate than the PRISM theory although still not in quantitative agreement with the simulations, especially at high densities. The dashed lines in Fig. 3(c) are predictions of the PRISM theory where the end sites are treated separately, and the 

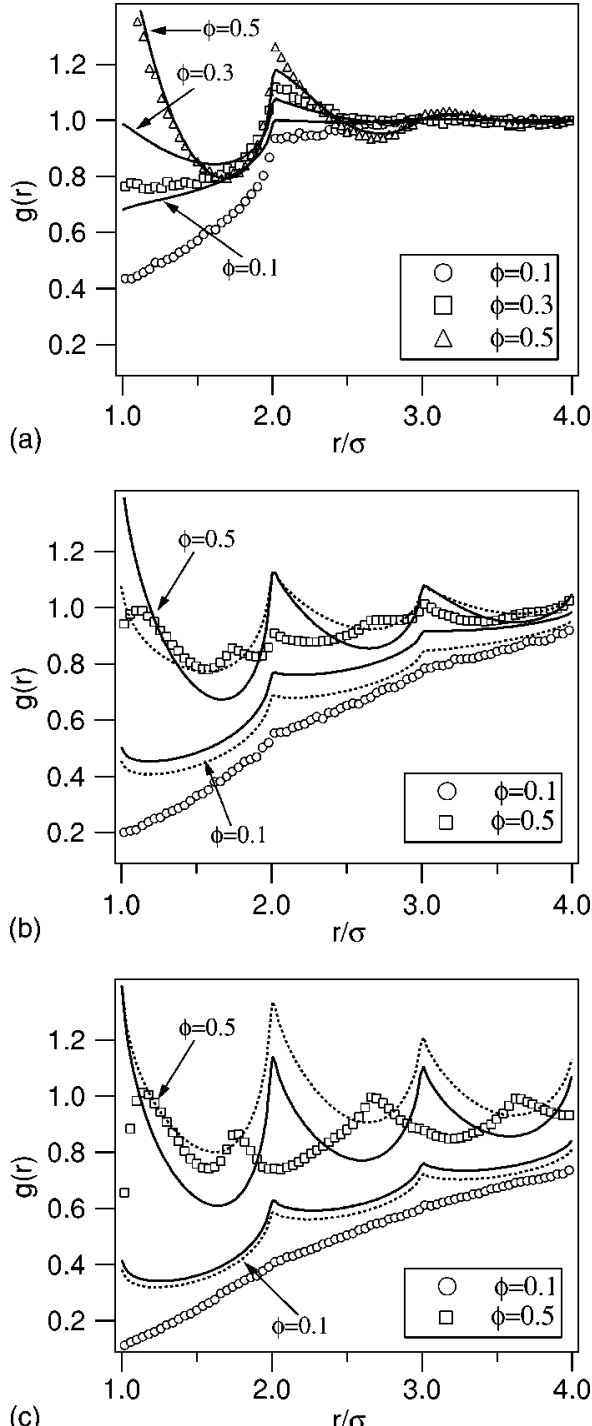

(c)

$r / \sigma$

FIG. 3. Comparison of theoretical predictions for $g(r)$ to Monte Carlo simulations (symbols) of tangent-disk rods for various area fractions (as marked) and for (a) $N=2$, (b) $N=4$, and (c) $N=8$. Solid lines are predictions of the PRISM theory where all sites are treated as equivalent. In (b) dashed lines are RISM predictions and in (c) dashed lines are PRISM predictions where the end sites are treated explicitly and the middle sites are treated as equivalent.

remaining (six) interior sites are treated as equivalent. Again there is an improvement in the predictions of the theory, compared to PRISM, although the difference in this case is less than what was seen in 4-mers. One can therefore expect that for long chains relaxing the equivalent site approximation will not result in a significant improvement in the PRISM predictions.

We attempt to establish why the performance of the theory is poorer in two dimensions than in three dimensions. One possibility is that the PY closure is not accurate and another is that the RISM equations are not "diagrammatically proper." We therefore consider the PRISM theory with the HNC closure, and a polymeric version of the diagrammatically proper Chandler-Silbey-Ladanyi (CSL) theory, ${ }^{14}$ with the PY closure. At low area fractions, e.g., $\phi \leqslant 0.3$ for $N=32$, the PRISM theory with the HNC closure does not converge. At high area fractions we are able to converge the

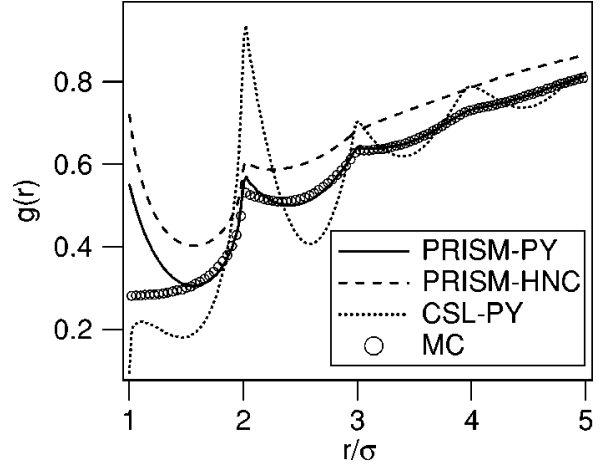

FIG. 4. Comparison of predictions of theories for $g(r)$ to Monte Carlo simulations (Ref. 3) (symbols) of flexible chains for $N=32$ and $\phi=0.5$.

theory, but the results are not as accurate as with the PY closure. The polymer-CSL theory, on the other hand, predicts unphysical negative values for $g(r)$ at low area fractions, and significantly overestimates the structure in $g(r)$ at high area fractions. Figure 4 compares predictions of the PRISM theory with PY and HNC closures, and the polymer-CSL theory with the PY closure for $N=32$ and $\phi=0.5$. For this area fraction, PRISM with the PY closure is quite accurate for $r>1.5 \sigma$. PRISM with the HNC closure overestimates the value of $g(r)$ but is otherwise similar to the PY closure. The diagrammatically proper polymer-CSL theory significantly overestimates the structure in $g(r)$ and is clearly the least accurate of the theories tested.

\section{CONCLUSIONS}

The PRISM theory for polymers is investigated for the pair correlation functions in melts of two-dimensional polymers. For flexible chains the theory, with the PY closure, significantly overestimates the value of the pair correlation function at short distances. For low densities the theory is not very accurate, but for high densities the theory is accurate for distances greater than about $1.5 \sigma$. Overall, the theory is not nearly as accurate as it is for freely-jointed chains in three dimensions. For fluids of dimers, the theory is accurate at high densities, but for longer rods the pair correlation functions predicted by the theory are qualitatively different from that seen in simulations, similar to what is seen in three dimensions. ${ }^{13}$ For short rods, removing the equivalent site approximations, i.e., using RISM instead of PRISM, results in more accurate predictions for $g(r)$ at low densities.

It is not clear why the PRISM theory displays such marked deficiencies for two-dimensional polymers. We find that the predictions of PRISM with the HNC closure, and the diagrammatically proper CSL theory (with the PY closure) are significantly worse than the PRISM/PY theory. Although the theory is unable to treat hidden sites, ${ }^{15}$ this cannot be the only reason because there are no hidden sites in melts of rods, for which the theory is not accurate either. In general, it is difficult to determine the reason for the failure of an integral equation theory. For example, it is not entirely clear why the PY theory is more accurate than the HNC for hard spheres, which the latter is more accurate for the restricted primitive model of electrolytes. 
It is also possible that the origin of the deficiencies in the theory are different for rods than for chains. For rods, the failure of the theory might be related to the strong orientational order in these systems, which cannot be captured by an approach based on RISM. For chains, the theory might fail for a different reason. Although the exact intramolecular correlations are input into the theory, this is an averaged function. In two-dimensional systems the conformational fluctuations are larger, at high densities, than in three-dimensional systems ${ }^{3}$ and it is possible that this is a source of the relatively poorer performance of the theory. This could be tested, in principle, by fashioning a model where conformational fluctuations can be controlled. This is a little tricky because one would have to restrict conformational changes without increasing orientational order (which excludes stiff chains) or otherwise changing the packing in a significant fashion.

The theory does appear to be more accurate when the structure of the fluid is similar to that of a fluid of hard disks. For example, the theory is quite accurate for dimers at high densities where the packing is similar in some respects to hard disks. And the theory is in qualitative error for rods, where the packing is very different from hard disks. In this case the "correlation hole" depletion effects are quite prominent at low densities, and local orientational order is quite prominent at high densities, as can be surmised from Fig. 1, and the predictions of the theory are poor, similar to what are found in three dimensions. In two-dimensional systems, the chains do not interpenetrate in the same fashion as in three dimensions, and depletion effects are important at all densities. The packing of chains in two dimensions is therefore quite different from that of disks. This might explain why the PRISM theory is more accurate in three dimensions than in two dimensions. In that sense it is a more dramatic manifestation of the shortcomings of the theory which are already present in more complex models of three-dimensional chains such as alkanes ${ }^{16}$ and vinyl polymers. ${ }^{17}$

We conclude that the liquid structure of two-dimensional polymers is an interesting problem for which popular integral equation theories with standard closure approximations are not accurate. It is possible that accurate predictions might be obtained via polymeric density functional theory, ${ }^{18}$ which for three-dimensional systems, is more accurate and internally consistent than the PRISM theory. This theory has the disadvantage, however, that the two molecule problem must be solved exactly, normally via exact enumeration or computer simulation. Although numerically intensive, this is a feasible and promising route to the structure of short chain polymers.

\section{ACKNOWLEDGMENT}

This material is based upon work supported by the National Science Foundation under Grant No. CHE-0315219.

\footnotetext{
${ }^{1}$ A. Yethiraj, Adv. Chem. Phys. 121, 89 (2002), and references therein.

${ }^{2}$ B. Maier and J. O. Radler, Phys. Rev. Lett. 82, 1911 (1999).

${ }^{3}$ A. Yethiraj, Macromolecules 36, 5854 (2003).

${ }^{4}$ P.-G. de Gennes, Scaling Concepts in Polymer Physics (Cornell University Press, Ithaca, 1979).

${ }^{5}$ J. des Cloizeaux and G. Jannink, Polymers in Solution (Oxford Science, Oxford, 1990).

${ }^{6}$ K. S. Schweizer and J. G. Curro, Phys. Rev. Lett. 58, 246 (1987); for a recent review see K. S. Schweizer and J. G. Curro, Adv. Polym. Sci. 116, 319 (1994); K. S. Schweizer, Adv. Chem. Phys. 98, 1 (1997).

${ }^{7}$ D. Chandler and H. C. Andersen, J. Chem. Phys. 57, 1930 (1972).

${ }^{8}$ E. F. David and K. S. Schweizer, J. Chem. Phys. 100, 7767 (1994); 100, 7784 (1994).

${ }^{9}$ B. J. Sung and A. Yethiraj, J. Chem. Phys. 119, 6916 (2003).

${ }^{10}$ A. Yethiraj and K. S. Schweizer, J. Chem. Phys. 97, 1455 (1992).

${ }^{11}$ F. Lado, J. Comput. Phys. 8, 417 (1971).

${ }^{12}$ M. A. Bates and D. Frenkel, J. Chem. Phys. 112, 10034 (2000).

${ }^{13}$ A. Yethiraj, R. Dickman, G. Szamel, M. L. Rosinberg, and E. Kierlik, Mol. Phys. 82, 937 (1994).

${ }^{14}$ D. Chandler, R. Silbey, and B. M. Ladanyi, Mol. Phys. 46, 1335 (1982).

${ }^{15}$ L. J. Lowden and D. Chandler, J. Chem. Phys. 61, 5228 (1974).

${ }^{16}$ A. Yethiraj, J. Chem. Phys. 102, 6874 (1995).

${ }^{17}$ A. Yethiraj, J. G. Curro, and J. J. Rajasekaran, J. Chem. Phys. 103, 2229 (1995).

${ }^{18}$ A. Yethiraj, H. Fynewever, and C.-Y. Shew, J. Chem. Phys. 114, 4323 (2001).
} 